\title{
Eine eigenständige Insomnieform: subjektiv nicht erholsamer Schlaf
}

\author{
Kann nicht erholsamer Nachtschlaf \\ unabhängig von Ein- und Durch- \\ schlafstörungen auftreten, und \\ welche Auswirkungen hat er auf die \\ Tagesbefindlichkeit?
}

- Patienten mit nicht organischer Insomnie leiden an Ein- und/oder Durchschlafstörungen, einem subjektiv nicht erholsamen Schlaf oder einer Kombination dieser Symptome. Diese schlafbezogenen Beschwerden führen meist zu einer erheblichen Beeinträchtigung der Tagesbefindlichkeit und Leistungsfähigkeit. Die Komponente der Nichterholsamkeit steht in der Praxis oft eher im Hintergrund. Dabei ist nicht erholsamer Schlaf keine seltene Beschwerde.

Die Studie wurde über einen Zeitraum von 98 Tagen an 28 Zentren zur Diagnostik von Schlafstörungen in den USA durchgeführt. Es handelt sich um ein querschnittsbasiertes Studiendesign, um Schlafstörungen und Tagesbeeinträchtigungen bei Patientengruppen mit nicht erholsamem Schlaf zu untersuchen, je nachdem ob gleichzeitig Einund Durchschlafstörungen vorlagen.

Insgesamt wurden 579 Patienten untersucht, die bereits drei Monate oder länger mindestens dreimal pro Woche beklagten, am Morgen nicht erholt zu erwachen. Die Probanden wurden nach ihren subjektiven Angaben in verschiedene Gruppen eingeteilt: Patienten mit Einschlafstörungen ( $\mathrm{n}=138)$, Durchschlafstörungen $(n=44)$, Einund Durchschlafstörungen $(\mathrm{n}=125)$ und ausschließlich nicht erholsamem Schlaf (keine Ein- oder Durchschlafstörung, $n=192$ ). Alle Probanden wurden zwei Nächte im Schlaflabor untersucht, um zu überprüfen, ob sich die berichtete Schlafstörung polysomnografisch objektivieren lässt

Einschlafstörungen konnten bei 56 der 138 Patienten objektiviert werden (41\%), Durchschlafstörungen bei 18/44 (41\%) und Ein- und Durchschlafstörungen bei 37/125 (30\%) und Aus-

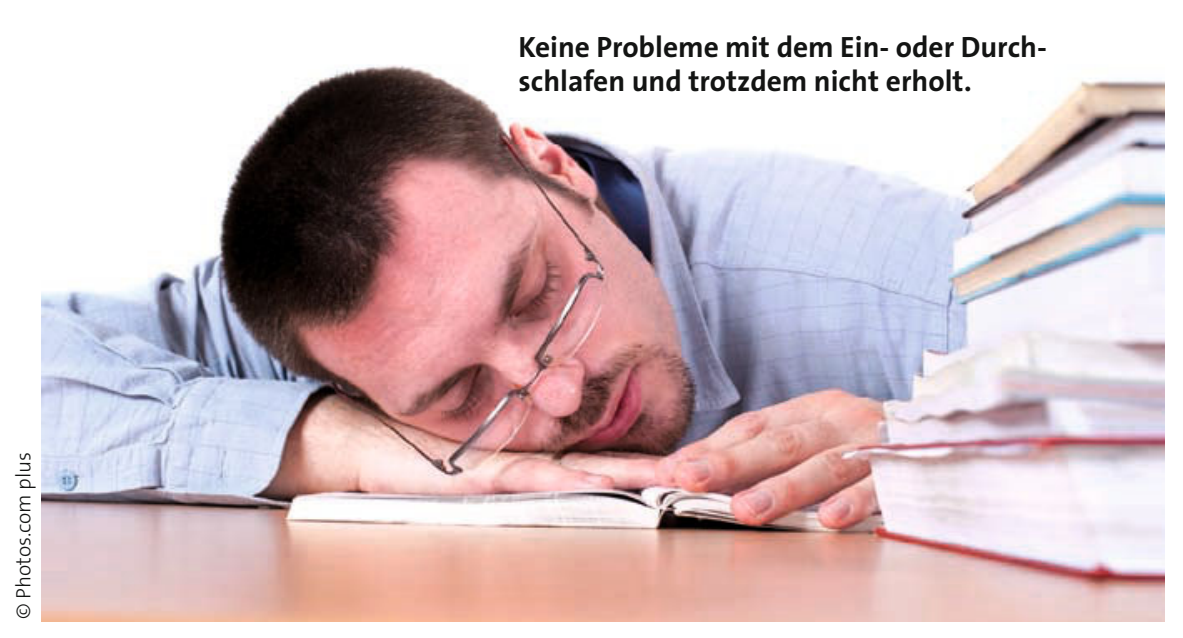

schluss einer Ein- oder Durchschlafgruppe bei subjektiv nicht erholsamem Nachtschlaf bei 115/192 (60\%). Bei den Gesunden (subjektiv keine Schlafbeschwerden) fand sich bei 52/80 (65\%) keine Ein- oder Durchschlafstörung in der PSG.

Es wurde eine explorative Analyse durchgeführt, um mögliche objektive Charakteristika von nicht erholsamem Schlaf zu identifizieren. Die Probanden mit nicht erholsamem Schlaf verbrachten, v. a. in der ersten Stunde der Nacht, 10,45 Minuten weniger in den Schlafstadien 3 und 4 (Tiefschlaf) und über die ganze Nacht etwas weniger Zeit im REM-Schlaf.

Die Fragebögen ergaben, dass Probanden mit nicht erholsamem Schlaf im Vergleich zu Gesunden signifikante Beeinträchtigungen in der Tagesbefindlichkeit (v. a. erhöhte Tagesmüdigkeit), der Leistungsfähigkeit und der Lebensqualität aufwiesen, die vergleichbar mit denjenigen Einschränkungen sind, an denen Patienten mit Ein- und/oder Durchschlafstörungen leiden.

\section{Kommentar}

Es handelt sich um eine hochrangig publizierte Studie, die multizentrisch und über die Dauer von drei Monaten durchgeführt wurde. Ziel war es, die Charakteristika von nicht erholsamem Schlaf als oft ver- nachlässigte Insomniekomponente zu untersuchen.

Bei den Ergebnissen wird erneut die in der schlafmedizinischen Praxis häufig zu beobachtende Diskrepanz zwischen subjektiv erlebter (Nicht-) Erholsamkeit und objektivierten Schlafparametern sichtbar. Das Schlafprofil der Patienten mit nicht erholsamem Schlaf stimmte weitestgehend mit dem der Gesunden überein, was die Einschlaflatenz und Gesamtschlafzeit betraf. Allein in der Analyse der in den einzelnen Schlafstadien verbrachten Zeit ergaben sich leichte Unterschiede. Als Schlussfolgerung für die Praxis sollte nicht erholsamer Nachtschlaf als Symptom ernst genommen werden, auch wenn vom Patienten keine weiteren Schlafbeschwerden berichtet werden. Bei der diagnostischen Abklärung ist der Ausschluss einer organischen Ursache der Nichterholsamkeit (z. B. Schlaf-Apnoe-Syndrom, Schlafmangelsyndrom, nächtliche periodische Beinbewegungen) mittels einer Polysomnografie wichtig sowie eine allgemeinmedizinische und psychiatrische Beurteilung zum Ausschluss körperlicher und psychiatrischer Ursachen.

V. HiRscher U. D. RiemanN, Freiburg I. BR. -

- T. Roth et al.

Nonrestorative sleep as a distinct component of insomnia. Sleep 33 (2010) 449-458 